\title{
Dietary Intake and Gastrointestinal Integrity in Runners Undertaking High-Intensity Exercise in the Heat
}

\author{
Naroa Etxebarria and Nicole A. Beard \\ University of Canberra
}

\author{
Alice Wallett \\ University of Canberra \\ and Australian Institute of Sport
}

\author{
Maree Gleeson \\ University of Newcastle Australia
}

Warren A. McDonald, Kate L. Pumpa,
and David B. Pyne
University of Canberra

\begin{abstract}
Gastrointestinal disturbances are one of the most common issues for endurance athletes during training and competition in the heat. The relationship between typical dietary intake or nutritional interventions and perturbations in or maintenance of gut integrity is unclear. Twelve well-trained male endurance athletes (peak oxygen consumption $=61.4 \pm 7.0 \mathrm{ml} \cdot \mathrm{kg}^{-1} \cdot \mathrm{min}^{-1}$ ) completed two trials in a randomized order in $35^{\circ} \mathrm{C}$ (heat) and $21{ }^{\circ} \mathrm{C}$ (thermoneutral) conditions and kept a detailed nutritional diary for eight consecutive days between the two trials. The treadmill running trials consisted of 15 min at $60 \%$ peak oxygen consumption, $15 \mathrm{~min}$ at $75 \%$ peak oxygen consumption, followed by $8 \times 1$-min high-intensity efforts. Venous blood samples were taken at the baseline, at the end of each of the three exercise stages, and $1 \mathrm{hr}$ postexercise to measure gut integrity and the permeability biomarker concentration for intestinal fatty-acid-binding protein, lipopolysaccharide, and lipopolysaccharidebinding protein. The runners self-reported gut symptoms $1 \mathrm{hr}$ postexercise and 3 days postexercise. The heat condition induced large (45-370\%) increases in intestinal fatty-acid-binding protein, lipopolysaccharide-binding protein, and lipopolysaccharide concentrations compared with the baseline, but induced mild gastrointestinal symptoms. Carbohydrate and polyunsaturated fat intake $24 \mathrm{hr}$ preexercise were associated with less lipopolysaccharide translocation. Protein, carbohydrate, total fat, and polyunsaturated fat intake ( 8 days) were positively associated with the percentage increase of intestinal fatty-acid-binding protein in both conditions (range of correlations, $95 \%$ confidence interval $=.62-.93[.02, .98]$ ). Typical nutrition intake partly explained increases in biomarkers and the attenuation of symptoms induced by moderate- and high-intensity exercise under both heat and thermoneutral conditions.
\end{abstract}

Keywords: endurance athletes, i-FABP, macronutrients

Endurance exercise in combination with heat exposure challenges many physiological functions, including the gastrointestinal (GI) system. GI discomfort can affect $>60 \%$ of individuals engaged in endurance events (Jeukendrup et al., 2000; Stuempfle,

(C) 2021 The Authors. Published by Human Kinetics, Inc. This is an Open Access article distributed under the terms of the Creative Commons Attribution-NonCommercial-NoDerivatives 4.0 International License, CC BY-NC-ND 4.0, which permits the copy and redistribution in any medium or format, provided it is not used for commercial purposes, no modifications are made, appropriate credit is given, and a link to the license is provided. See http://creativecommons.org/licenses/ by-nc-nd/4.0. This license does not cover any third-party material that may appear with permission in the article. For commercial use, permission should be requested from Human Kinetics, Inc., through the Copyright Clearance Center (http://www. copyright.com).

Etxebarria, Wallett, Pumpa, and Pyne are with the Research Institute for Sport and Exercise, University of Canberra, Canberra, Australia. Etxebarria is also with the University Drive, Bruce, Australian Capital Territory, Australia. Beard is with the Faculty of Science and Technology, University of Canberra, Canberra, Australia. Gleeson is with the Faculty of Health and Medicine, University of Newcastle, Newcastle, Australia. Wallett is also with the Australian Institute of Sport, Bruce, Australian Capital Territory, Australia. McDonald is with Rugby Australia and the Faculty of Health, University of Canberra, Canberra, Australia. Etxebarria (naroa. etxebarria@canberra.edu.au) is corresponding author.
Hoffman, \& Hew-Butler, 2013), which can negatively affect exercise, training, and competitive performance. Exercising in the heat is a common practice for endurance athletes and can compromise epithelial integrity, consequently, increasing intestinal permeability and endotoxemia (Costa et al., 2017). Most GI disturbances occur on race day (Stuempfle et al., 2013) or longduration ( $>2 \mathrm{hr}$ ) exercise at moderate intensity (approximately $60 \%$ peak oxygen consumption $\left[\mathrm{VO}_{2}\right.$ peak]; Snipe et al., 2018). However, less is known about GI dysregulation during higher intensity interval training $(<1 \mathrm{hr})$ completed during the preparation months prior to a major competition.

Recent nutritional interventions aiming to ameliorate GI disturbances have mainly focused on nutritional intake during exercise in the heat. Ingestion of carbohydrates and protein during prolonged exercise can limit disturbances to gut epithelial integrity and small intestine permeability (Snipe et al., 2017). While acute nutritional interventions during exercise may help, it is unclear how or whether an individual's typical nutritional intake affects their susceptibility to GI disturbances during exercise in the heat. This study aimed to explore whether an athlete's typical nutritional intake influences any GI disturbance induced by high-intensity exercise in the heat. This information could assist sports dietitians in advising athletes during preparation for training and competition in hot conditions. 


\section{Methods}

\section{Experimental Design}

A randomized crossover trial was used to characterize the typical dietary intake and disturbances to GI integrity after submaximal and maximal intensity exercise in hot and temperate conditions. An 8-day food diary was kept by each participant. GI integrity was determined by measurement of serum biomarkers. The study was conducted during the winter, and none of the participants were heat acclimated or acclimatized. The study was approved by the Human Research Ethics Committee at the University of Canberra (HREC 17-117) in accordance with the Declaration of Helsinki, and all participants provided written informed consent.

\section{Participants}

Twelve well-trained male runners $(60 \pm 20 \mathrm{~km}$ of weekly running) participated in this study (age $=37 \pm 7$ years; body mass $=72.5 \pm$ $8.1 \mathrm{~kg} ; \mathrm{VO}_{2}$ peak $=61.4 \pm 7.0 \mathrm{ml} \cdot \mathrm{kg}^{-1} \cdot \mathrm{min}^{-1}$; height $\left.=179 \pm 5 \mathrm{~cm}\right)$. All participants completed a prestudy health screening questionnaire, including a gut-health questionnaire, and were deemed healthy, injury-free, and asymptomatic for any major gut disturbances at the time of the study. One of the participants had Type 1 diabetes and was a vegetarian.

\section{Typical Nutritional Intake}

Daily nutritional intake was recorded by the participants over a period of 8 consecutive days between the two trials, starting $24 \mathrm{hr}$ prior to the first trial and finishing $2 \mathrm{hr}$ before completing the second trial. This period was assumed to be representative of the participants' habitual nutritional intake. The participants were provided with a food diary and instructed to record all food and beverages (including quantities) consumed over the 8 days. The data from the food diaries were entered into the Easy Diet Diary mobile application and analyzed using FoodWorks (version 10; Xyris Pty Ltd, Brisbane, Australia). Relative $\left(\mathrm{kg}^{-1}\right)$ macronutrient consumption, energy intake, and diet quality were assessed according to the Australian Dietary Guidelines (National Health and Medical Research Council, 2006). The participants replicated their nutritional intake during the $24 \mathrm{hr}$ prior to each intervention trial and avoided food for $2 \mathrm{hr}$ prior to exercising.

\section{Exercise Protocols}

The athletes reported to the laboratory wearing appropriate clothing and footwear for running. The $\mathrm{VO}_{2}$ peak test, consisting of submaximal steady-state stages, followed by a graded progressive maximal test, was undertaken a week prior to "heat" and "thermoneutral" interventions. The intervention exercise protocol replicated a typical training session for endurance athletes (a combination of steady-state and high-intensity running intervals) to facilitate the transfer of the outcomes into regular training. The participants were assigned to either heat $\left(35{ }^{\circ} \mathrm{C}, 75 \%\right.$ relative humidity) or thermoneutral $\left(18{ }^{\circ} \mathrm{C}, 30 \%\right.$ relative humidity) environment conditions in a randomized and counter-balanced manner, completed a week apart. Each exercise intervention included two submaximal 15-min sustained efforts and a maximal high-intensity interval section. The submaximal runs were completed at $60 \%$ and $75 \% \mathrm{VO}_{2}$ peak, as determined during the preexperimental $\mathrm{VO}_{2}$ peak test, with a 5-min break in between. After a 5-min recovery break, the maximal high- intensity interval section consisted of $8 \times 1$-min efforts $\left(95 \% \mathrm{VO}_{2}\right.$ peak), with 1 min of passive recovery in between or until volitional exhaustion. All testing sessions were conducted on a motorized treadmill (Pulsar, Nußdorf, Germany) in an environmental heat chamber. Hydration status was checked using a digital urine SG refractometer (DUR-PEN; ATAGO, Tokyo, Japan), and a value of 1.030 was deemed to be the highest threshold of hydration for exercising. If the participants exceeded this value, they were encouraged to drink $200-300 \mathrm{ml}$ over a period of $30 \mathrm{~min}$ in order to improve their hydration status.

\section{GI Perceptual Measures and Biomarkers}

A gut comfort questionnaire (15 questions) completed prior to testing and $72 \mathrm{hr}$ post each trial was used to rate the frequency of GI discomfort from 0 (never) to 4 (all the time) and adverse symptoms (West et al., 2013). The participants also completed a 1-hr postexercise GI discomfort questionnaire (a subset of eight questions) adapted from the one used at the baseline (Likert scale 0-4), with a higher number indicating increased GI disturbance. Hydration status was measured prior to each trial by Urine Specific Gravity (ATAGO), and the participants were permitted to drink water ad libitum during exercise, with no food consumed. The participants provided $5 \times 10-\mathrm{ml}$ blood samples for each intervention: at the baseline, at the end of the first and second 15-min steady-state runs, after the $8 \times 1$-min maximal high-intensity intervals, and at $1 \mathrm{hr}$ postexercise. All baseline measures were taken at a thermoneutral temperature $\left(21^{\circ} \mathrm{C}\right)$. Blood samples were drawn and handled as previously described (Wallett et al., 2020). The enzyme-linked immunosorbent assays for biomarkers, which represent GI permeability, included lipopolysaccharide (LPS), lipopolysaccharidebinding protein, and intestinal fatty-acid-binding protein (iFABP). All biomarkers were analyzed in duplicate, as previously described (Wallett et al., 2020).

\section{Statistical Analysis}

The descriptive data are presented as mean $\pm S D$. Within- and between-participant variability in the GI biomarkers are reported as the percentage coefficient of variation. The magnitude of change between the standardized means (effect size) was interpreted against the following criteria: $<0.2$ as trivial, $0.2-0.6$ as small, $0.6-1.2$ as moderate, $1.2-2.0$ as large, and $>2.0$ as very large. The precision of estimation was determined using $90 \%$ confidence limits. When the magnitude of the standardized effect crossed the threshold of a small positive and small negative $( \pm 0.2)$, the change or difference was deemed unclear. Pearson correlation analysis with $95 \%$ confidence limits was used to measure the association between daily nutritional intake and GI responses to exercise using the following criteria: $<.1$ as trivial, .1-.3 as small, $.3-.5$ as moderate, and .5-.7 as large and extended (Cohen, 1988), to include $.7-.9$ as very large and $>.9$ as nearly perfect.

\section{Results}

All participants were well hydrated before each intervention with mean Urine Specific Gravity values for the heat condition under the accepted $<1.03$ threshold. Water ingestion during exercise was $570 \pm 500 \mathrm{ml}$ for heat and $300 \pm 290 \mathrm{ml}$ for thermoneutral conditions. During the heat condition, only $5 \pm 3$ maximal intensity intervals were completed (due to exhaustion) versus $7 \pm 2$ intervals completed during the thermoneutral condition. 


\section{Nutritional Status}

The athletes' mean daily energy intake was $10,700 \pm 2,700 \mathrm{~kJ}$, $85 \pm 25 \%$ of their estimated daily energy requirement $(12,800 \pm$ $1,200 \mathrm{~kJ})$. The macronutrient intake is shown in Table 1 . The athletes met the minimum requirements for daily protein consumption for endurance athletes of 1.6-2 $\mathrm{g} \cdot \mathrm{kg}^{-1} \cdot \mathrm{day}^{-1}$ (Burke \& Deakin, 2009). However, their carbohydrate intake was below the expected threshold for those undertaking moderate exercise $(5-7 \mathrm{~g} / \mathrm{kg})$ and substantially lower than recommended for heavy training days (7-12 g/kg; Burke $\&$ Deakin, 2009). The quality of the daily nutritional intake, represented as recommended servings, revealed a high intake of grains $(11 \pm 4 / 6)$ and only half the recommended vegetable intake $(3 \pm 2 / 6)$. Fruit, protein, and dairy intake were close to the recommended serves (2, 3, and 2.5 serve, respectively).

The acute nutritional intake during the 24 -hr period preceding the two exercise trials was consistently and positively associated with increases in i-FABP, especially for the thermoneutral condition (Figure 1a). In contrast, a higher intake of carbohydrates prior to the heat trial and protein and polyunsaturated fat intake prior to the thermoneutral trial were related to lower LPS concentrations (Figure 1b). The total energy intake in the $24 \mathrm{hr}$ prior to the thermoneutral trial was very largely associated with exerciseinduced changes in i-FABP and its recovery $1 \mathrm{hr}$ post (range of correlations, $95 \%$ confidence interval $=r$ : .71-.90 $[.19, .98])$, with unclear associations in the heat trial.

Only changes in i-FABP in response to exercise were associated with the 8-day nutritional intake, primarily with total fat, protein, polyunsaturated fat, and, to a lesser extent, carbohydrates (Figure 2). The correlations were greatest in the higher intensity exercise phases for both the heat and thermoneutral interventions. During moderate-intensity exercise, nutrition was mainly related to changes in i-FABP during the thermoneutral trial, while only the elevated i-FABP levels postheat were related to nutritional intake. Diet quality (dairy, fruit, and vegetables) showed mainly unclear associations with changes in GI biomarkers. A lower mean energy intake was associated with a greater recovery of i-FABP $1 \mathrm{hr}$ post the thermoneutral condition $(r=.74[.16, .94])$.

\section{Thermal Conditions}

Biomarker responses in individuals to exercise showed considerable between-subject variability, particularly in the heat intervention (Figure 3). Heat exposure induced larger increases in GI biomarkers from the baseline $(45-370 \%)$ than observed in the thermoneutral condition (Figure 3). The i-FABP and lipopolysaccharide-binding protein concentrations failed to return to baseline levels $1 \mathrm{hr}$ postexercise in the heat intervention trial.

\section{GI Perceptual Measures}

The different responses in biomarkers between the trials were not mirrored with changes in self-reported GI symptoms neither $1 \mathrm{hr}$ postexercise nor $72 \mathrm{hr}$ postexercise. Both trials induced similar low levels of self-reported GI symptoms in these well-trained runners, with an overall GI discomfort score of $2.1 \pm 1.8 / 32$ (mean $\pm S D$, /total score of 32 for acute GI symptoms) for heat and 1.4 $\pm 1.9 / 32$ after thermoneutral $1 \mathrm{hr}$ postexercise. Feeling nauseous was the main symptom reported in both trials. During the 3 days after the trials, the runners self-reported a GI discomfort score of $4.8 \pm 3.5 /$ 60 for heat and $5.1 \pm 2.9 / 60$ after thermoneutral, well below the preexercise screening scores of $12.3 \pm 2.6 / 60$. Increased frequency of bowel movements and hunger were the main symptoms reported $72 \mathrm{hr}$ post exercise trials. Nutritional intake was not associated with decreased acute symptoms $1 \mathrm{hr}$ postexercise, while starch, fiber, polyunsaturated fat, and total fat were positively associated with $1 \mathrm{hr}$ postexercise symptoms $(r=.57-.66[.09, .89])$. In contrast, there was a moderate and inverse or negative association between the symptoms reported $72 \mathrm{hr}$ postexercise and both the protein intake ( $24 \mathrm{hr}$ pre-exercise and also the 8-day period) and carbohydrate intake in the $24 \mathrm{hr}$ leading up to the thermoneutral trial $(r=-.55$ to $-.61[.05,-.88])$.

\section{Discussion}

Variable associations between the GI biomarker, GI symptoms, and nutritional intake suggest they are not tightly coupled. Instead,

\section{Table 1 Breakdown of the Macronutrients $(\mathrm{g} / \mathrm{kg})$ During the 8-Day Detailed Reporting of the Nutritional Intake Completed via the Easy Diet Diary Mobile Application}

\begin{tabular}{|c|c|c|c|c|c|c|c|c|}
\hline $\begin{array}{l}\text { \#Number of } \\
\text { runners }\end{array}$ & Protein & $\begin{array}{c}\text { Total } \\
\text { fat }\end{array}$ & $\begin{array}{c}\text { Saturated } \\
\text { fat }\end{array}$ & Trans fat & $\begin{array}{c}\text { Polyunsaturated } \\
\text { fat }\end{array}$ & Carbohydrates & Starch & Fiber \\
\hline 1 & $1.2 \pm 0.3$ & $1.1 \pm 0.3$ & $0.4 \pm 0.1$ & $0.02 \pm 0.01$ & $0.16 \pm 0.06$ & $3.6 \pm 0.8$ & $1.9 \pm 0.7$ & $0.3 \pm 0.1$ \\
\hline 2 & $1.0 \pm 0.3$ & $0.8 \pm 0.6$ & $0.2 \pm 0.2$ & $0.01 \pm 0.02$ & $0.13 \pm 0.10$ & $3.0 \pm 1.0$ & $2.3 \pm 1.0$ & $0.3 \pm 0.1$ \\
\hline 3 & $1.3 \pm 0.5$ & $1.0 \pm 0.4$ & $0.4 \pm 0.2$ & $0.02 \pm 0.02$ & $0.13 \pm 0.06$ & $2.6 \pm 1.4$ & $1.4 \pm 1.1$ & $0.4 \pm 0.1$ \\
\hline 4 & $1.4 \pm 0.2$ & $1.3 \pm 0.3$ & $0.5 \pm 0.1$ & $0.02 \pm 0.01$ & $0.21 \pm 0.06$ & $5.5 \pm 1.4$ & $3.6 \pm 0.7$ & $0.6 \pm 0.2$ \\
\hline 5 & $1.5 \pm 0.3$ & $1.1 \pm 0.4$ & $0.4 \pm 0.2$ & $0.02 \pm 0.01$ & $0.20 \pm 0.13$ & $3.8 \pm 1.4$ & $2.6 \pm 1.2$ & $0.5 \pm 0.1$ \\
\hline 6 & $1.3 \pm 0.6$ & $0.6 \pm 0.3$ & $0.3 \pm 0.2$ & $0.01 \pm 0.01$ & $0.08 \pm 0.05$ & $2.2 \pm 1.1$ & $1.3 \pm 0.6$ & $0.3 \pm 0.1$ \\
\hline 7 & $1.7 \pm 0.3$ & $0.8 \pm 0.3$ & $0.3 \pm 0.1$ & $0.01 \pm 0.00$ & $0.10 \pm 0.06$ & $3.4 \pm 0.9$ & $1.9 \pm 0.5$ & $0.3 \pm 0.1$ \\
\hline 8 & $1.6 \pm 0.5$ & $1.9 \pm 0.8$ & $0.8 \pm 0.3$ & $0.05 \pm 0.03$ & $0.23 \pm 0.12$ & $5.7 \pm 0.8$ & $3.7 \pm 0.7$ & $0.5 \pm 0.2$ \\
\hline 9 & $1.2 \pm 0.3$ & $1.1 \pm 0.4$ & $0.3 \pm 0.1$ & $0.01 \pm 0.01$ & $0.26 \pm 0.11$ & $4.6 \pm 0.9$ & $2.9 \pm 0.6$ & $0.6 \pm 0.2$ \\
\hline 10 & $2.0 \pm 0.3$ & $1.3 \pm 0.3$ & $0.5 \pm 0.2$ & $0.02 \pm 0.01$ & $0.21 \pm 0.05$ & $3.7 \pm 0.6$ & $2.4 \pm 0.4$ & $0.4 \pm 0.1$ \\
\hline 11 & $2.6 \pm 0.3$ & $2.5 \pm 0.9$ & $1.1 \pm 0.5$ & $0.04 \pm 0.02$ & $0.26 \pm 0.06$ & $7.0 \pm 0.7$ & $4.2 \pm 0.5$ & $0.6 \pm 0.1$ \\
\hline 12 & $2.5 \pm 1.1$ & $1.9 \pm 0.9$ & $0.6 \pm 0.4$ & $0.02 \pm 0.02$ & $0.42 \pm 0.16$ & $4.3 \pm 1.4$ & $2.6 \pm 1.1$ & $0.6 \pm 0.1$ \\
\hline Total & $\begin{array}{c}1.6 \pm 0.5 \\
(1.6-2.0)^{*}\end{array}$ & $1.3 \pm 0.5$ & $0.5 \pm 0.3$ & $0.02 \pm 0.01$ & $0.20 \pm 0.09$ & $\begin{array}{c}4.1 \pm 1.4 \\
(5-12)^{*}\end{array}$ & $2.6 \pm 0.9$ & $\begin{array}{c}0.5 \pm 0.1 \\
30 \text { (g/day)* }\end{array}$ \\
\hline
\end{tabular}

Note. Descriptive values are presented as mean $\pm S D$.

*Recommended intake (Burke \& Deakin, 2009). 


\section{$75 \% \mathrm{VO}_{2}$ peak}

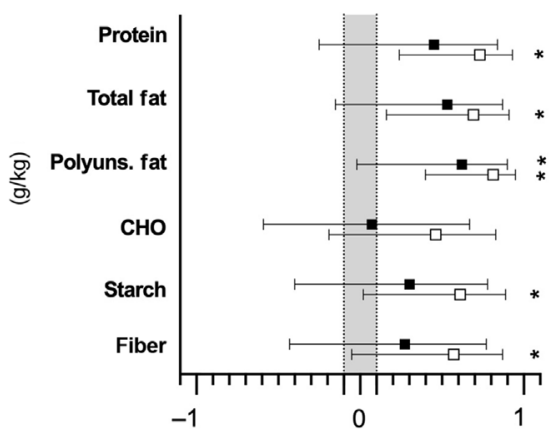

Maximal efforts

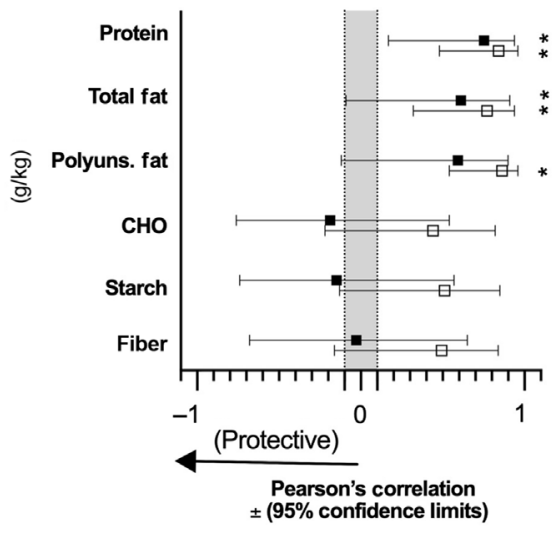

(b) LPS

- Heat
$60 \% \mathrm{VO}_{2}$ peak

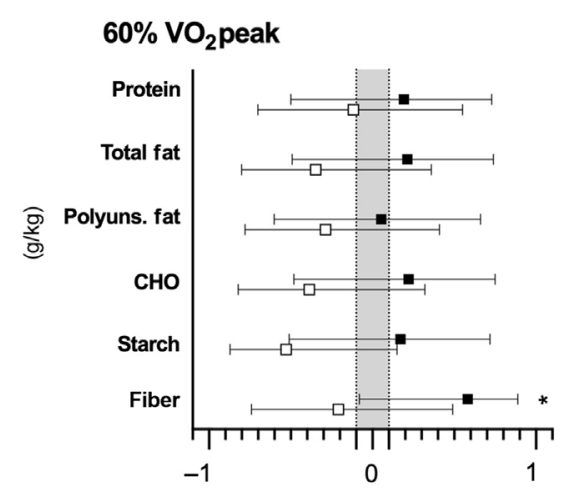

Maximal efforts

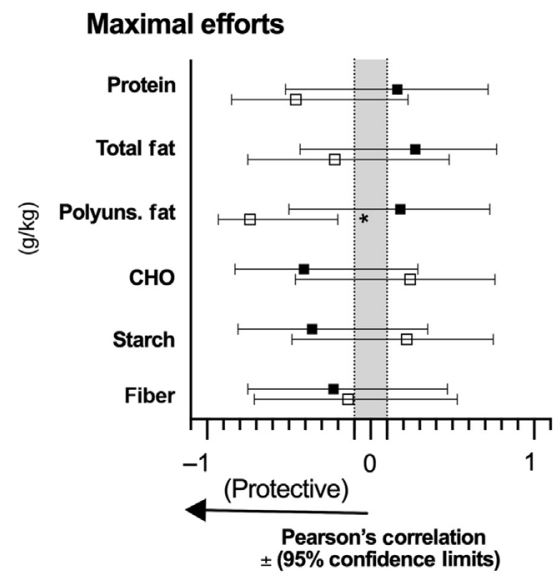

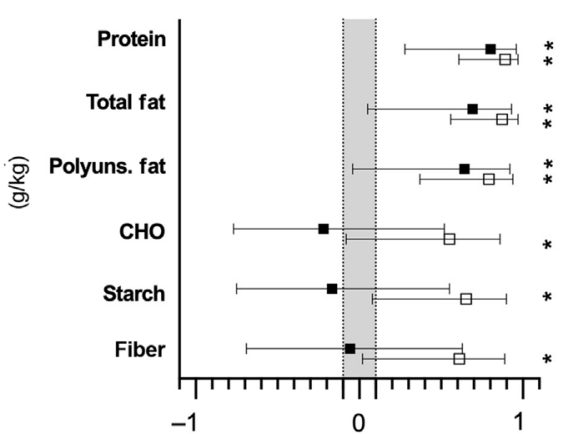

$1 \mathrm{hr}$ post

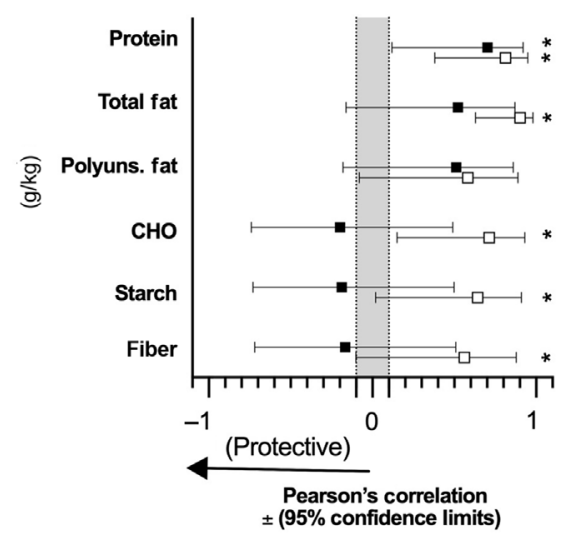

- Thermoneutral
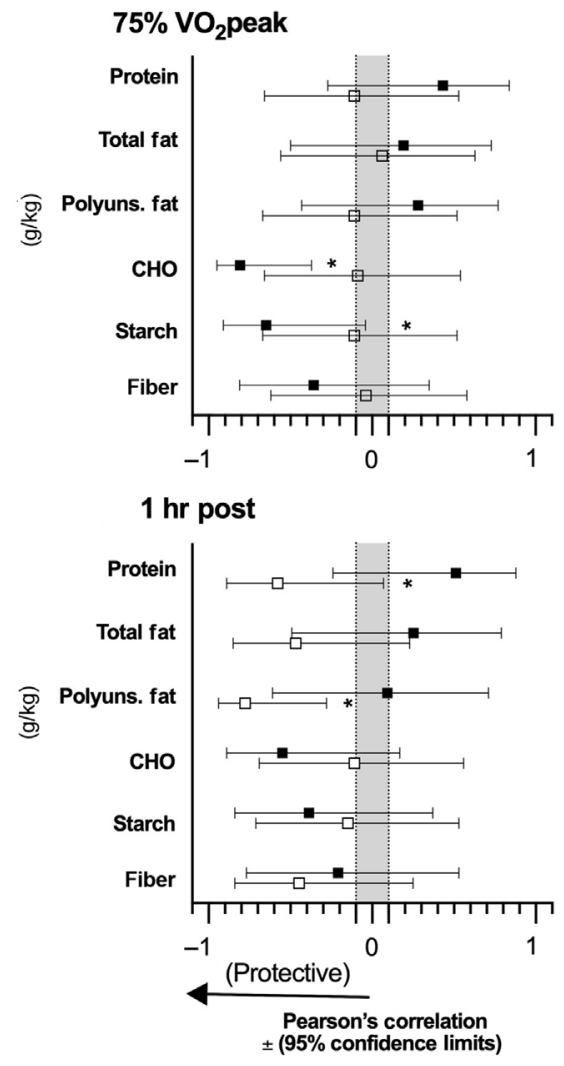

Figure 1 - Correlation ( $\pm 95 \%$ confidence limits) between typical nutritional intake ( $24 \mathrm{hr}$ preexercise) and \%change in (a) circulatory (i-FABP) and (b) LPS from baseline to $60 \% \mathrm{VO}_{2}$ peak exercise, $75 \% \mathrm{VO}_{2}$ peak exercise, high-intensity efforts, and 1 hr postexercise for both heat and thermoneutral conditions. A correlation was deemed clear $(*)$ when the confidence interval did not extend past both -0.1 and 0.1 thresholds (shaded area). Magnitude of the correlation was interpreted against the following criteria: $<0.1$ as trivial, $0.1-0.3$ as small, $0.3-0.5$ as moderate, $0.5-0.7$ as large, $0.7-0.9$ as very large, and $>0.9$ as near perfect. i-FABP $=$ intestinal fatty-acid-binding protein; $\mathrm{LPS}=$ lipopolysaccharide $; \mathrm{CHO}=$ carbohydrate; Polyuns. fat $=$ polyunsaturated fat; $\mathrm{VO}_{2}$ peak $=$ peak oxygen consumption. 


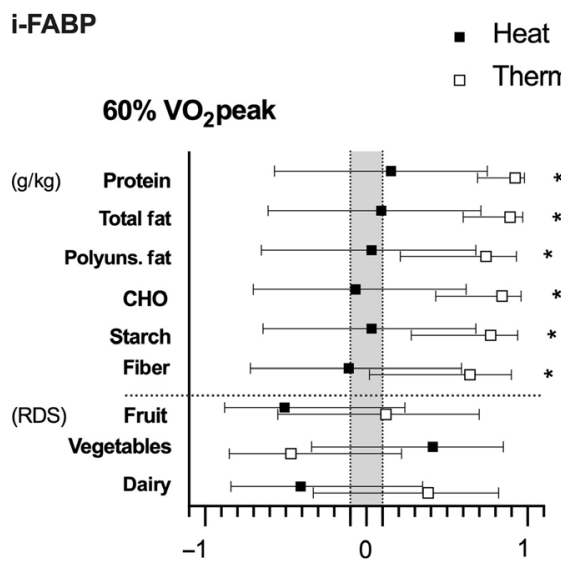

Heat

Thermoneutral
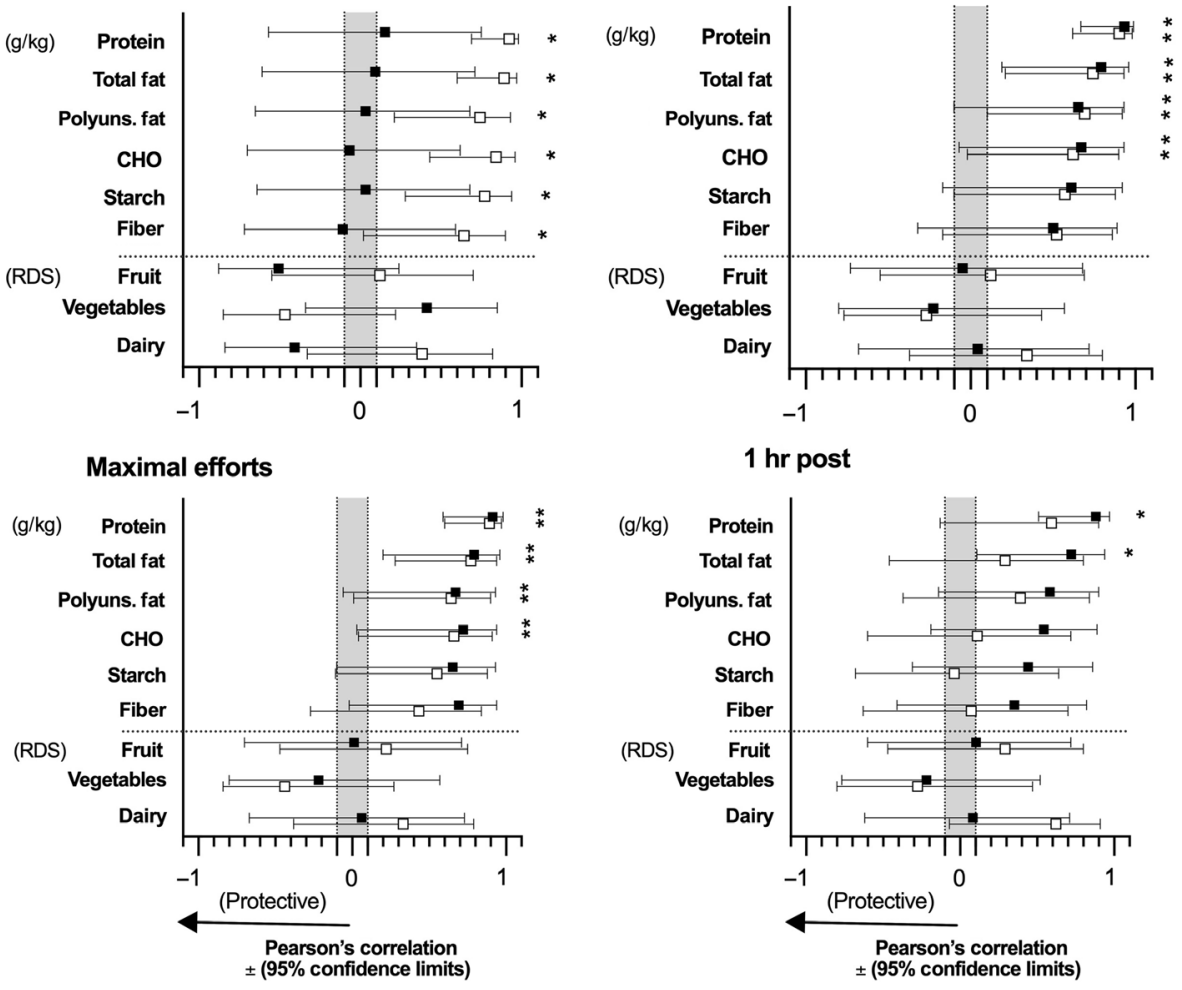

Figure 2 - Correlation ( $\pm 95 \%$ confidence limits) between typical nutritional intake (8-day period) and $\%$ change in circulatory [i-FABP] from baseline to $60 \% \mathrm{VO}_{2}$ peak exercise, $75 \% \mathrm{VO}_{2}$ peak exercise, high-intensity efforts, and $1 \mathrm{hr}$ postexercise for both heat and thermoneutral conditions. A correlation was deemed clear $\left(^{*}\right)$ when the confidence interval did not extend past both -0.1 and 0.1 thresholds (shaded area). Magnitude of the correlation was interpreted against the following criteria: $<.1$ as trivial, .1-.3 as small, .3-.5 as moderate, .5-.7 as large, .7-.9 as very large, and >.9 as near perfect. $\mathrm{i}-\mathrm{FABP}=$ intestinal fatty acid-binding protein; $\mathrm{CHO}=$ carbohydrate; Polyuns. fat = polyunsaturated fat; $\mathrm{VO}_{2}$ peak $=$ peak oxygen consumption; $\mathrm{RDS}=$ recommended daily serve

they are indicative of modest associations between GI permeability integrity, nutrition, and discomfort symptoms. Typical nutritional intake that meets nutritional guidelines does not appear to play a significant role in reducing an exercise-induced GI disturbance during high-intensity exercise in both cool and hot conditions. However, higher carbohydrate and polyunsaturated fat intake in the $24 \mathrm{hr}$ prior to exercising in the heat might play a role in minimizing LPS translocation into the circulatory system, although this observation was not consistent throughout the exercise bout. Higher protein and carbohydrate intake in the $24 \mathrm{hr}$ prior to the thermoneutral trial also showed a potential protective role against GI symptoms in the days after the trial. However, longer term or mean weekly nutritional intake did not have a protective role against the changes in GI integrity, measured by representative biomarkers, during high-intensity exercise in the heat.

The average ( 8 days) nutritional intake was not associated with a positive effect in reducing high-intensity exercise-induced increases in i-FABP. The high-intensity exercise performed in a hot environment elicited modest exercise-induced dysregulation of GI integrity compared with thermoneutral conditions. The failure to return to baseline $1 \mathrm{hr}$ postexercise in the heat might have negative implications for runners undertaking multiple sessions in a day. The modest increase in GI disturbances, reflected by increased biomarkers during exercise, was not associated with a marked increase in self-reported symptoms. Collectively, the data indicate that the GI system of trained runners may have adapted to the stress induced by exercise and heat exposure and/or that the level of increase in the biomarkers we observed has minimal clinical significance in healthy and fit runners.

The majority of evidence on the beneficial nutrient intake that ameliorates exercise-induced GI dysregulation is associated with macronutrient intake during exercise, triggering postprandial hyperemia, blunting GI perturbations by improving splanchnic perfusion, or increasing blood flow to the GI tract (van Wijck et al., 2012). Frequent intake of both protein and carbohydrates during exercise may decrease GI disturbances, with carbohydrate intake reducing symptoms and increasing endotoxin clearance (Snipe et al., 2017). We showed a substantial positive correlation between carbohydrate intake and increase in i-FABP, despite the lower than recommended carbohydrate intake. In the absence of nutrient intake immediately prior to or during exercise, the benefits of protein and carbohydrate intake during exercise inducing postprandial hyperemia would dissipate.

Our results indicate that the increased response of i-FABP during exercise across the range of intensities is positively associated with certain macronutrients. The i-FABP is involved in the intracellular solubilization of and cytosolic reservoir for fatty acids (Haunerland \& Spener, 2004). The positive correlation between 

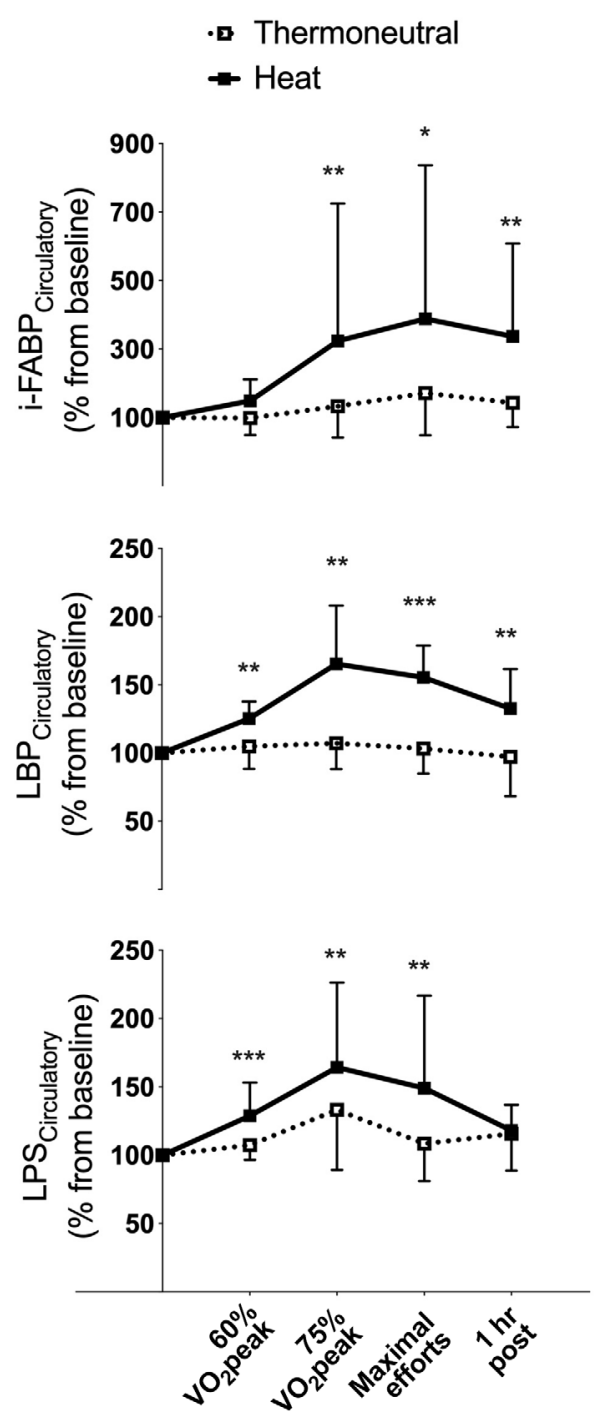

Figure 3 - Responses of gastrointestinal biomarkers to both environmental conditions (heat and thermoneutral); LPS, LBP, and i-FABP. Substantial differences in the mean change between baseline and the rest of the time-points between heat and thermoneutral. The different time-points represented are as follows: Pre = baseline; Ex. $1=15 \mathrm{~min}$ at $60 \% \mathrm{VO}_{2}$ peak; Ex. $2=15 \mathrm{~min}$ at $75 \% \mathrm{VO}_{2}$ peak; $\mathrm{Max}=$ end of maximal intervals; and $1 \mathrm{hr}$ Post $=1 \mathrm{hr}$ postexercise. The magnitude of standardized change in means (effect size) was interpreted against the following criteria: $<0.2$ as trivial, $0.2-0.6$ as small, $0.6-1.2$ as moderate, $1.2-2.0$ as large, and $>2.0$ as very large. $\mathrm{VO}_{2}$ peak = peak oxygen consumption; $\mathrm{LPS}=$ lipopolysaccharide; $\mathrm{LBP}=$ lipopolysaccharide-binding protein; $\mathrm{i}-\mathrm{FABP}=$ intestinal fatty acidbinding protein. Magnitude of the standardized difference is represented as follows: *small; **moderate; ***large.

polyunsaturated and total fat and i-FABP might be evidence of a higher concentration (or cycling) of i-FABP stored in the cell. Upon enterocyte injury, the protein rapidly diffuses through the interstitial space into circulation (van Wijck et al., 2014). Consequently, a higher concentration of circulatory i-FABP observed in individuals with a higher total fat intake may not be as a result of greater enterocyte injury, but a higher concentration of i-FABP per cell damaged (longer term adaptation from a higher total fat nutritional intake) that diffuses to the circulatory system. Regardless, the short half-life of $\mathrm{i}-\mathrm{FABP}$ in plasma (approximately $11 \mathrm{~min}$ ) implies that the i-FABP increase we observed is in response to exercise, as the participants did not ingest any food $2 \mathrm{hr}$ prior to exercise and during exercise. Taken together, the data suggest that the daily nutritional intake of runners might not have a protective effect but does not aggravate exercise-induced GI symptoms in either thermoneutral or heat conditions.

It would appear that the lipopolysaccharide-binding protein may not be the best GI biomarker to use to progress knowledge in this area. It is possible that water consumption during exercise impacted the GI integrity biomarkers. Dehydration affects GI function (Kartaram et al., 2019; van Nieuwenhoven et al., 2000), and the participants ingested different amounts of fluids in both trials. Although we ensured they were adequately hydrated prior to commencing exercise, the end-of-exercise hydration status was (unfortunately) not recorded, which might have yielded additional insights. Future work should focus on the daily dietary provision to minimize the shortcomings of self-reporting nutritional intake and investigate the short-term ingestion of nutrients before and during exercise.

In this exploratory study, we observed a strong association between the selected macronutrients ingested and injury to gut integrity, with limited evidence that a balanced typical nutritional intake protects against GI disturbance during exercise in the heat. However, future research should address the nutritional manipulation of macronutrient intake in the day or hours leading to a highintensity exercise bout, as we observed some protective associations with GI disturbances. In the current study, the runners ingested a well-balanced nutritional intake and met the recommended sports nutrition guidelines prior to the exercise. However, it is unclear whether manipulating the diet (such as carbohydrate loading) prior to an endurance event would offer protective benefits, comparable to ingesting carbohydrates during exercise, against exercise-induced GI perturbations. The increased dietary carbohydrate in the $24 \mathrm{hr}$ leading up to exercising in the heat could have a protective effect against LPS translocation. Future work should evaluate the dietary manipulation of macronutrients in the days and hours leading to strenuous exercise in the heat and how, for example, increased carbohydrate intake might affect/ameliorate GI disturbances and symptoms. Future research should explore the close dynamics between the acute ( $24 \mathrm{hr}$ preexercise) manipulation of macronutrients, GI function, and exercise.

\section{Conclusion}

In the absence of nutritional intake during exercise, an athlete's typical dietary intake explains, in part, the increases in i-FABP induced by moderate sustained and high-intensity interval exercise $(<1 \mathrm{hr})$ in the heat.

\section{References}

Burke, L.M., \& Deakin, V. (2009). Clinical sports nutrition (5th ed.). Sydney, Australia: McGraw-Hill.

Cohen, J. (1988). Statistical power analysis for the behavioral sciences. In L. Erlbaum (Ed.), (2nd ed., p. 567). Hillsdale (NJ).

Costa, R.J.S., Miall, A., Khoo, A., Rauch, C., Snipe, R., Camoes-Costa, V., \& Gibson, P. (2017). Gut-training: The impact of two weeks repetitive gut-challenge during exercise on gastrointestinal status, glucose availability, fuel kinetics, and running performance. Applied Physiology, Nutrition, and Metabolism, 42(5), 547-557. PubMed ID: 28177715 doi:10.1139/apnm-2016-0453 
Haunerland, N.H., \& Spener, F. (2004). Fatty acid-binding proteinsinsights from genetic manipulations. Progress in Lipid Research, 43(4), 328-349. PubMed ID: 15234551 doi:10.1016/j.plipres.2004. 05.001

Jeukendrup, A.E., Vet-Joop, K., Sturk, A., Stegen, J.H., Senden, J., Saris, W.H., \& Wagenmakers, A.J. (2000). Relationship between gastrointestinal complaints and endotoxaemia, cytokine release and the acute-phase reaction during and after a long-distance triathlon in highly trained men. Clinical science (London), 98(1), 47-55. Retrieved from https://www.ncbi.nlm.nih.gov/pubmed/10600658

Kartaram, S., Mensink, M., Teunis, M., Schoen, E., Witte, G., Janssen Duijghuijsen, L., ... van Norren, K. (2019). Plasma citrulline concentration, a marker for intestinal functionality, reflects exercise intensity in healthy young men. Clinical Nutrition, 38(5), 2251-2258. PubMed ID: 30340895 doi:10.1016/j.clnu.2018.09.029

National Health and Medical Research Council. (2006). Nutrient reference values for Australia and New Zealand, including recommended dietary intakes. Retrieved from http://www.nhmrc.gov.au/guidelines/ publications/n35-n36-n37

Snipe, R.M.J., Khoo, A., Kitic, C.M., Gibson, P.R., \& Costa, R.J.S. (2017). Carbohydrate and protein intake during exertional heat stress ameliorates intestinal epithelial injury and small intestine permeability. Applied Physiology, Nutrition, and Metabolism, 42(12), 12831292. PubMed ID: 28777927 doi:10.1139/apnm-2017-0361

Snipe, R.M.J., Khoo, A., Kitic, C.M., Gibson, P.R., \& Costa, R.J.S. (2018). The impact of exertional-heat stress on gastrointestinal integrity, gastrointestinal symptoms, systemic endotoxin and cytokine profile. European Journal of Applied Physiology, 118(2), 389-400. PubMed ID: 29234915 doi:10.1007/s00421-017-3781-z
Stuempfle, K.J., Hoffman, M.D., \& Hew-Butler, T. (2013). Association of gastrointestinal distress in ultramarathoners with race diet. International Journal of Sport Nutrition and Exercise Metabolism, 23(2), 103-109. PubMed ID: 23006626 doi:10.1123/ijsnem.23.2.103

van Nieuwenhoven, M.A., Vriens, B.E., Brummer, R.J., \& Brouns, F. (2000). Effect of dehydration on gastrointestinal function at rest and during exercise in humans. European Journal of Applied Physiology, 83(6), 578-584. PubMed ID: 11192068 doi:10.1007/s004210000305

van Wijck, K., Lenaerts, K., Grootjans, J., Wijnands, K.A., Poeze, M., van Loon, L.J., ... Buurman, W.A. (2012). Physiology and pathophysiology of splanchnic hypoperfusion and intestinal injury during exercise: Strategies for evaluation and prevention. American Journal of Physiology-Gastrointestinal, 303(2), G155-G168. doi:10.1152/ ajpgi.00066.2012

van Wijck, K., Wijnands, K.A., Meesters, D.M., Boonen, B., van Loon, L.J., Buurman, W. A., ... Poeze, M. (2014). L-citrulline improves splanchnic perfusion and reduces gut injury during exercise. Medicine \& Science in Sports \& Exercise, 46(11), 2039-2046. PubMed ID: 24621960 doi:10.1249/MSS.0000000000000332

Wallett, A., Etxebarria, N., Beard, N., Saunders, P., Welvaert, M., Périard, J., ... Pyne, D. (2020). Running at increasing intensities in the heat induces transient gut perturbations. International Journal of Sports Physiology and Performance,1-7. PubMed ID: 33361496 doi:10. 1123/ijspp.2019-0973

West, N.P., Christophersen, C.T., Pyne, D.B., Cripps, A.W., Conlon, M.A., Topping, D.L., .. . Clarke, J.M. (2013). Butyrylated starch increases colonic butyrate concentration but has limited effects on immunity in healthy physically active individuals. Exercise Immunology Review, 19, 102-119. Retrieved from https://www.ncbi.nlm.nih.gov/pubmed/ 